\title{
Reduction of the effect of actuator saturation with periodic servo-constraints
}

\author{
László Bencsik, László L. Kovács \\ MTA-BME Research Group on Dynamics of Machines and Vehicles \\ Budapest, Hungary \\ \{bencsik, kovacs\}@mm.bme.hu \\ This manuscript is identical with published version.
}

\begin{abstract}
Saturation is an undesired event in trajectory tracking control of mechanical systems. When the actuators of a robotic device saturate the solution of the inverse dynamics problem cannot fully be realized, which results in deviations from the desired trajectory and loss of performance. It is generally hard to consider the limited actuator torques and the corresponding nonlinear effects in the control design. The most common way to handle the problem is recalculating the control forces and trying to adjust the desired trajectory such that saturation will not happen. In contrast we propose a switched control approach, where, upon saturation, a periodically switched input is applied to keep the reference point of the robot on the desired trajectory. For this, the desired motion is formulated by means of servo-constraints, and the periodic switching of these constraints is adjusted according to the variation of a new, manipulability type performance measure. It will be demonstrated that the proposed adaptive controller can reduce the undesired effects of actuator saturation effectively. An example is provided to show the application of the method and to compare the results with those of other methods taken from the literature.
\end{abstract}

keywords : Computed torque control, actuator saturation, periodic servo-constraints, manipulability measure, multibody dynamics 


\section{INTRODUCTION}

Actuator saturation occurs when the controller's output exceeds the physical limit of the actuators of a system. In this case the controller can no longer properly realize the desired motion, and as a result, large overshoots and sustained oscillation may develop. These increase the settling time and potentially can lead to an unstable motion.

In structured, industrial environments saturation can be prevented by careful trajectory planning based on the well defined task and operational conditions. The use of large, powerful actuators is often also an option. When these are not possible, e.g., in field and service robotics [1] where the environmental conditions and the task are a priory unknown, saturation can result in poor dynamic performance.

Performance deterioration resulting form saturation is often called actuator windup [2], and the control methods trying to minimize the undesired effects of saturation are commonly referred to as anti-windup techniques $[3,4,5]$. These techniques were originally developed for linear single-input

single-output systems [6]. A possible generalization to non-linear multi-input multi-output systems is shown in [7] by using partial feedback linearization. A method that is directly applicable to non-linear mechanical systems is presented in [8].

For trajectory tracking of robots, feedback linearization essentially gives the same control forces/torques as the classical computed-torque control method. Therefore, it is clear that when saturation happens, feedback linearization cannot cancel all the nonlinear dynamic terms [7] and the robot will deviate from the desired trajectory. A common element of the anti-windup control schemes is that the saturating control force is compared to the ideal control force, and based on this, the input of the controller is modified to reduce trajectory following error.

Instead, in our approach we aim to redistribute the load of the saturating actuator(s) among those which are still functioning in their nominal operating range. This is feasible in robotic systems where typically only a few, but different actuators saturate as the robot follows the commanded end-point 
trajectory. Often there are also more actuators available than what the task would require. For example, when cooperating robots are moving an object [9]. Still, depending on the number of saturated actuators, these robots can loose their ability to realize the desired task without redistributing the load on the actuators. In case of a single robot with as many actuators as the task requires, saturation will limit the control capabilities similar to those of the trivially underactuated system with fewer actuators than degrees-of-freedom.

In reference [10], upon saturation the controlled robotic system is considered temporarily underactuated, and a servo-constraint based controller $[11,12,13]$ is implemented to distribute the load on the actuators differently with and without actuator saturation. In the saturating phase, a reduced, linearly combined set of servo-constraints is used to minimize the trajectory following error. This is somewhat similar to the method proposed in [7], where the control force is recalculated by preserving the direction of the control action without saturation.

The present paper introduces a periodically switched controller which not only reduces the number of servo-constraints, but also adapts to the dynamics of the system during saturation. The periodic switching makes it possible to regulate all the original servo constraint over time, one after the other, and it also helps to stabilize the internal dynamics of the temporarily underactuated system [14]. It will also be demonstrated that a new performances measure, similar to dynamic manipulability, can effectively be used to algorithmically choose and update the periodic control input. 


\section{DYNAMIC FORMULATION}

\subsection{Equation of motion}

In modelling the dynamics of complex multibody systems the use of a non-minimum set, descriptor type coordinates is quite common [15]. In this case, the general form of the equation of motion is

$$
\begin{aligned}
\mathbf{M} \ddot{\mathbf{q}}+\boldsymbol{\Phi}^{\top} \boldsymbol{\lambda}+\mathbf{c} & =\mathbf{Q}+\mathbf{H u}, \\
\phi(\mathbf{q}, t) & =\mathbf{0}
\end{aligned}
$$

where $\mathbf{q}$ is the array of $n$ dependent coordinates, and $\phi$ collects the $m$ geometric constraints associated with the coordinates selected. Matrix $\mathbf{M}$ is the mass matrix, $\mathbf{\Phi}(\mathbf{q}, t)=\partial \boldsymbol{\phi} / \partial \mathbf{q}$ is the $m \times n$ Jacobian of the constraints, and $\boldsymbol{\lambda}$ is the array of Lagrangian multipliers corresponding to the constraints. Array $\mathbf{c}(\mathbf{q}, \dot{\mathbf{q}}, t)$ contains the Coriolis and centrifugal terms and $\mathbf{Q}(\mathbf{q})$ denotes external

forces other than the control inputs, such as gravity. The forces/torques of the actuators are represented by the $l \times 1$ array $\mathbf{u}$ and $\mathbf{H}(\mathbf{q})$ is the $n \times l$ control input matrix.

Equations (1) and (2) form a differential algebraic system of equations (DAE) with differentiation index 3. There are direct methods for solving these equations, but very often the method of Lagrange multipliers is used [15]. This method reduces the differentiation index to 1 by expressing the constraints at the acceleration level as

$$
\ddot{\phi}=\Phi \ddot{\mathbf{q}}+\dot{\Phi} \dot{\mathbf{q}}+\dot{\varphi}
$$

where $\varphi=\partial \phi / \partial t$, and makes it possible to express the equation of motion in the form

$$
\left[\begin{array}{cc}
\mathbf{M} & \boldsymbol{\Phi}^{\top} \\
\mathbf{\Phi} & 0
\end{array}\right]\left[\begin{array}{c}
\ddot{\mathbf{q}} \\
\lambda
\end{array}\right]=\left[\begin{array}{c}
\mathbf{Q}-\mathbf{c}+\mathbf{H u} \\
-\dot{\Phi} \dot{\mathbf{q}}-\dot{\varphi}
\end{array}\right]
$$


From Eq. (4) the accelerations $\ddot{\mathbf{q}}$ and the Lagrange multipliers $\boldsymbol{\lambda}$ can be computed, and the integration for $\mathbf{q}$ can be carried out by a suitable method (e.g., trapezoidal rule).

\subsection{Motion specification and inverse dynamics}

One can define the desired trajectory of a robot, by adding a second set of constraint equations, the so-called servo - or actuator constraints, to equations (1) and (2) [11, 16]. These are mathematically equivalent to the geometric constraints, however, the fact that these do not belong to physical interconnections of bodies makes an important difference. The servo-constraints, for example can describe the tool-center-point (TCP) trajectory of the robot, and the violation of these constraints is proportional to the tracking error. The violation of the geometric constraints is a measure of the accuracy of the numerical simulation only.

Servo-constraints represent also a useful tool for solving the inverse dynamics. This can be done similarly to the solution of Eq. (4) for the Lagrangian multipliers. In order to show this, let us consider the servo-constraints in the general form

$$
\gamma(\mathbf{q}, t)=\mathbf{p}(\mathbf{q})-\mathbf{h}(t)=\mathbf{0}
$$

where $\mathbf{p}(\mathbf{q})$ describes the output, e.g., position and orientation of the TCP of a robot, as function of the generalized coordinates, and $\mathbf{h}(t)$ is the desired output with explicit time dependence. The servo constraints can also be expressed at the acceleration level as

$$
\ddot{\gamma}=\Gamma \ddot{\mathbf{q}}+\dot{\Gamma} \dot{\mathbf{q}}-\dot{\mathrm{g}}
$$

where $\boldsymbol{\Gamma}=\partial \mathbf{p} / \partial \mathbf{q}$ is the Jacobian associated with the servo-constraints and $\mathbf{g}=\partial \mathbf{h} / \partial t$. Also, the control input $\mathbf{u}$ plays a similar role as the Lagrangian multipliers associated with the geometric constraints. It enforces the servo-constraints just like the physical structure of a system enforces the 
geometric constraints through the development of constraint forces described by Lagrangian multipliers. Therefore Eqs. (1), (3) and (6) can be combined in the compact form

$$
\left[\begin{array}{ccc}
\mathbf{M} & \boldsymbol{\Phi}^{\top} & -\mathbf{H} \\
\boldsymbol{\Phi} & 0 & 0 \\
\Gamma & 0 & 0
\end{array}\right]\left[\begin{array}{c}
\ddot{\mathbf{q}} \\
\lambda \\
\mathbf{u}
\end{array}\right]=\left[\begin{array}{c}
\mathrm{Q}-\mathbf{c} \\
-\dot{\boldsymbol{\Phi}} \dot{\mathbf{q}}-\dot{\varphi} \\
-\dot{\Gamma} \dot{\mathbf{q}}-\dot{\mathrm{g}}-\eta
\end{array}\right]
$$

where $\boldsymbol{\eta}(\mathbf{q})$ is a feedback control term. When $\boldsymbol{\eta}=\mathbf{0}$, then the solution of the system in (7) gives the control force $\mathbf{u}$ for the desired motion defined by the acceleration level servo-constraints. When the calculated control force is used in a feed-forward loop, this is called the computed torque control (CTC) method. To compensate for disturbances and modelling errors the feedback term may be chosen as $\boldsymbol{\eta}=K_{D} \dot{\gamma}+K_{P} \gamma$, where $K_{P}$ and $K_{D}$ are the proportional and derivative control gains, respectively. This control action is similar to the Baumgarte stabilization [17] widely used in dynamic simulation of multibody systems.

Given a motion specification, Eq. (7) can be solved for the required control efforts as long as there are enough non-saturating actuators to realize the desired motion. This is true for most of the fully actuated mechanical systems, but underactuated systems and systems with higher relative degree may require a special treatment $[11,18]$. When one or more actuators saturate, the solution of Eq.

(7) does not provide a feasible control force. Then only a subset of the original servo-constraints can be enforced by the remaining non-saturating actuators, and the system becomes temporarily underactuated with respect to the original task.

\section{PERIODIC SERVO CONSTRAINTS}

The basic problem in implementing a computed torque controller for systems with input torque saturation is that some states of the system will evolve uncontrollably during saturation. The dynamics associated with these uncontrolled states is referred to as the internal dynamics of the 


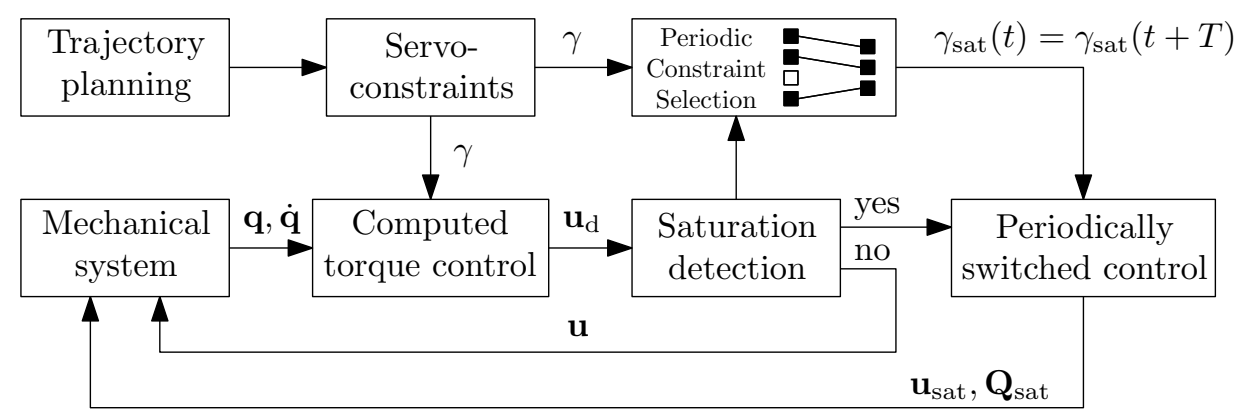

Figure 1: Block diagram of the proposed controller

system. It depends not only on the physical properties and the load of a system, but also on the selection of the controlled variables. Also, it can be unstable in which case the utmost control objective is to find a stabilizing controller. This may be achieved by slightly changing the controlled reference point by modifying the servo-constraints, or altering the structural properties of a robotic device $[12,11,13]$.

Considering sufficiently strong dynamic coupling, a different approach is to implement a switching controller that switches between two or more sets of servo constraints such that the internal dynamics remains stable and the desired trajectory is realized within tolerances. The switching can be tied to certain events or done periodically with time. In the followings, we will use periodic feedback, the stabilization effect of which is well known in case of time delay system [19]. These systems has infinitely many poles, but only a few actuators.

A periodically switched controller was successfully applied for the trajectory tracking of an underactuated service robot in our previous work [14]. It that paper the switching pattern and period were determined based on numerical stability analysis and simulations which makes the online implementation difficult. Instead, here we propose a method which rely only on the actual state of the system, and adjusts the parameters of the periodically switched controller continuously on the course of operation.

The general idea is outlined in Fig. 1. It shows that until saturation occurs the classical computed 
torque controller is applied. Then, the number of servo-constraints is appropriately reduced, different sets of servo constraints are formed, and these are combined into a new periodic servo-constraint signal $\gamma_{\mathrm{sat}}(t)=\gamma_{\mathrm{sat}}(t+T)$ with period $T$. One possibility to do this is to take the linear combination

$$
\gamma_{\mathrm{sat}}=\sum_{i=1}^{N} \alpha_{i} \widehat{\gamma}_{i} \quad \text { with } \quad \alpha_{i}(t)=\alpha_{i}(t+T)
$$

where array $\widehat{\gamma}_{i}$ contains a reduced set of servo-constraints with $r<l$ elements, coefficients $\alpha_{i}$ control the switching between the different sets of servo constraints, and $N<\left(\begin{array}{l}l \\ r\end{array}\right)$ is the number of the considered different sets. Figure 1 also shows that during saturation a reduced size, $r \times 1$, control input $\mathbf{u}_{\text {sat }}$ is considered and the effect of the saturated actuators is represented by the constant external force term $\mathbf{Q}_{\text {sat }}$. With these, and using the subscript sat to denote the terms affected by saturation, equation (7) can be rewritten as

$$
\left[\begin{array}{ccc}
\mathbf{M} & \boldsymbol{\Phi}^{\top} & -\mathbf{H}_{\mathrm{sat}} \\
\mathbf{\Phi} & \mathbf{0} & \mathbf{0} \\
\boldsymbol{\Gamma}_{\mathrm{sat}} & \mathbf{0} & \mathbf{0}
\end{array}\right]\left[\begin{array}{c}
\ddot{\mathbf{q}} \\
\boldsymbol{\lambda} \\
\mathbf{u}_{\mathrm{sat}}
\end{array}\right]=\left[\begin{array}{c}
\mathbf{Q}+\mathbf{Q}_{\mathrm{sat}}-\mathbf{c} \\
-\dot{\boldsymbol{\Phi}} \dot{\mathbf{q}}-\dot{\varphi} \\
-\dot{\boldsymbol{\Gamma}}_{\mathrm{sat}} \dot{\mathbf{q}}-\dot{\mathrm{g}}_{\mathrm{sat}}-K_{D} \dot{\boldsymbol{\gamma}}_{\mathrm{sat}}-K_{P} \gamma_{\mathrm{sat}}
\end{array}\right]
$$

For a given reduced set of servo-constraints $\gamma_{\text {sat }}$, the required control inputs $\mathbf{u}_{\text {sat }}$ can be calculated

based on this equation. For the selection and switching between different, reduced sets of servo-constraints, a manipulability type performance index is proposed. The performance index is evaluated at certain time instants and a periodic control signal is formed such that more time is allocated for the sets with the higher indices. This way, those servo-constraint sets are prioritized where there is a stronger coupling between the control inputs and the desired accelerations. The proposed performance index can be introduced by considering the minimum coordinate parametrization of the equation of motion in (9) as

$$
\mathbf{B}^{\top} \mathbf{M B} \ddot{\mathbf{p}}+\mathbf{M} \dot{\mathbf{B}} \dot{\mathbf{p}}+\mathbf{B}^{\top}\left(\mathbf{c}-\mathbf{Q}-\mathbf{Q}_{\mathrm{sat}}\right)=\mathbf{B}^{\top} \mathbf{H}_{\mathrm{sat}} \mathbf{u}_{\mathrm{sat}}
$$


where transformation $\dot{\mathbf{q}}=\mathbf{B} \dot{\mathbf{p}}$ defines the new parametrization. Note, that $\mathbf{p}$ is conveniently selected as the array of operational space coordinates, the evolution of which are directly prescribed by the servo-constraints. Then, neglecting external forces other than the control input, the linearised equations become

$$
\overline{\mathbf{M}}\left(\ddot{\mathbf{p}}-\overline{\mathbf{M}}^{-1} \mathbf{B}^{\top} \mathbf{Q}_{\mathrm{sat}}\right)=\mathbf{B}^{\top} \mathbf{H}_{\mathrm{sat}} \mathbf{u}_{\mathrm{sat}},
$$

where $\overline{\mathbf{M}}=\mathbf{B}^{\top} \mathbf{M B}$ is the operational space mass matrix [20], and $\ddot{\mathbf{p}}-\overline{\mathbf{M}}^{-1} \mathbf{B}^{\top} \mathbf{Q}_{\text {sat }}$ is the acceleration due to the non-saturated actuators. By denoting this acceleration with $\overline{\mathbf{a}}$ and introducing $\mathbf{G}=\mathbf{B}^{\top} \mathbf{H}_{\text {sat }}$, the uniformity of the control input to acceleration gain is given by the manipulability ellipsoid

$$
\overline{\mathbf{a}}^{\top} \mathcal{M} \overline{\mathbf{a}} \leq 1 \quad \text { with } \quad \mathcal{M}=\left(\mathbf{G}^{\dagger} \overline{\mathbf{M}}\right)^{\top}\left(\mathbf{G}^{\dagger} \overline{\mathbf{M}}\right)
$$

where $\mathbf{G}^{\dagger}$ is the Moore-Penrose pseudo-inverse of $\mathbf{G}$. Also, let $\mathbf{e}_{i}$ be an $l$ dimensional unit vector, containing $r$ non-zero elements associated with a certain reduced set of servo-constraints. With this, the effect of the control input in a specific direction can be characterized by the performance index

$$
z_{i}=\frac{1}{\sqrt{\mathbf{e}_{i}^{\top} \mathcal{M} \mathbf{e}_{i}}},
$$

and the time to be devoted to the realization of the considered set of servo-constraints within one control period may be obtained as

$$
\Delta t_{i}=\frac{z_{i} T}{\sum_{j=1}^{N} z_{j}}
$$

Equations (13) and (14) help to find a physically motivated periodic control signal. This needs to be updated in the course of the motion by considering the current state of the system, or, in off-line 




Figure 2: Two-link planar manipulator

calculations, the desired values of the servo-constraints.

\section{EXAMPLE}

In the following, as the simplest non-trivial example, we consider the trajectory tracking control of a two-link manipulator. This is similar to the anti-windup control problem of a SCARA robot analyised in [8].

The system shown in Fig. 2 has two identical links with $l_{1}=l_{2}=0.4 \mathrm{~m}$ and $m_{1}=m_{2}=0.2 \mathrm{~kg}$. A homogeneous mass distribution is considered, and the configuration is described by the independent generalized coordinates $\mathbf{q}=\left[\theta_{1}, \theta_{2}\right]^{\top}$. The manipulator is in the horizontal plane, and its TCP (tool-center-point) is required to follow a circular trajectory with a trapezoidal velocity profile. This is shown in Fig. 3 where the base joint of the manipulator is located in the origin, and the circular trajectory is centered at $x=0$ and $y=0$. The maximum values of the acceleration and velocity for the trapezoidal velocity profile are $a_{\max }=0.7 \mathrm{~m} / \mathrm{s}^{2}$ and $v_{\max }=0.7 \mathrm{~m} / \mathrm{s}$. The corresponding operational space trajectories, $x_{D}(t)$ and $y_{D}(t)$, are shown in Fig. 3. The saturation limit of the shoulder motor is $\tau_{1 \max }=0.6 \mathrm{Nm}$, and it is assumed that the second actuator, $\tau_{2}$, will not saturate. In addition, parameters $K_{P}$ and $K_{D}$ in (9) are set to $40 \mathrm{~N} / \mathrm{m}$ and $30 \mathrm{Ns} / \mathrm{m}$, respectively. 
For the considered simple manipulator it is straightforward to derive the equation of motion in operational space coordinates $\mathbf{p}=[x, y]^{\top}$. Because of the initially chosen independent set of relative coordinates $\mathbf{q}=\left[\theta_{1}, \theta_{2}\right]^{\top}$, there is no need for geometric constraints, and the transformation matrix $\mathbf{B}$ in (10) is simply the inverse of the manipulator Jacobian. Also, for input $\mathbf{u}=\left[\tau_{1}, \tau_{2}\right]^{\top}$, the control input matrix $\mathbf{H}$ is the identity matrix. Taking these into consideration, and assuming that only the shoulder motor with the higher loads will saturate, the components of the linearized equation of

$$
\overline{\mathbf{M}}=\left[\begin{array}{cc}
\bar{m}_{11} & \bar{m}_{12} \\
\bar{m}_{21} & \bar{m}_{22}
\end{array}\right], \mathbf{B}=\left[\begin{array}{cc}
b_{11} & b_{12} \\
b_{21} & b_{22}
\end{array}\right], \ddot{\mathbf{p}}=\left[\begin{array}{c}
\ddot{x} \\
\ddot{y}
\end{array}\right], \mathbf{Q}_{\mathrm{sat}}=\left[\begin{array}{c}
\tau_{1 \max } \\
0
\end{array}\right], \mathbf{H}_{\mathrm{sat}}=\left[\begin{array}{l}
0 \\
1
\end{array}\right] \text { and } \mathbf{u}_{\mathrm{sat}}=\tau_{2},
$$

where, for the sake of brevity, the elements of $\overline{\mathbf{M}}$ and $\mathbf{B}$ are only indicated by single symbols. In addition, the circular trajectory shown in Fig. 3 is defined by the servo-constraints

$$
\gamma(\mathbf{p}(\mathbf{q}), \mathbf{t})=\left[\begin{array}{l}
l_{1} \cos \left(\theta_{1}\right)+l_{2} \cos \left(\theta_{1}+\theta_{2}\right) \\
l_{1} \sin \left(\theta_{1}\right)+l_{2} \sin \left(\theta_{1}+\theta_{2}\right)
\end{array}\right]-\left[\begin{array}{c}
x_{D} \\
y_{D}
\end{array}\right]
$$

where $x_{D}(t)$ and $y_{D}(t)$ define the desired motion of the TCP of the manipulator. Note, that in the acceleration level equation (6) matrix $\boldsymbol{\Gamma}=\partial \mathbf{p} / \partial \mathbf{q}$ is precisely the manipulator Jacobian. It can be also seen that, during saturation, only the remaining single actuator, $\tau_{2}$, can be used to realize both servo-constraints.

By having a planar motions specification, one can either consider $\gamma_{1}$ or $\gamma_{2}$ as single elements of two different reduced sets of servo-constraints. According to (8), these different sets can be combined in a single expression as

$$
\gamma_{\mathrm{sat}}=\alpha \widehat{\gamma}_{1}+(1-\alpha) \widehat{\gamma}_{2}
$$



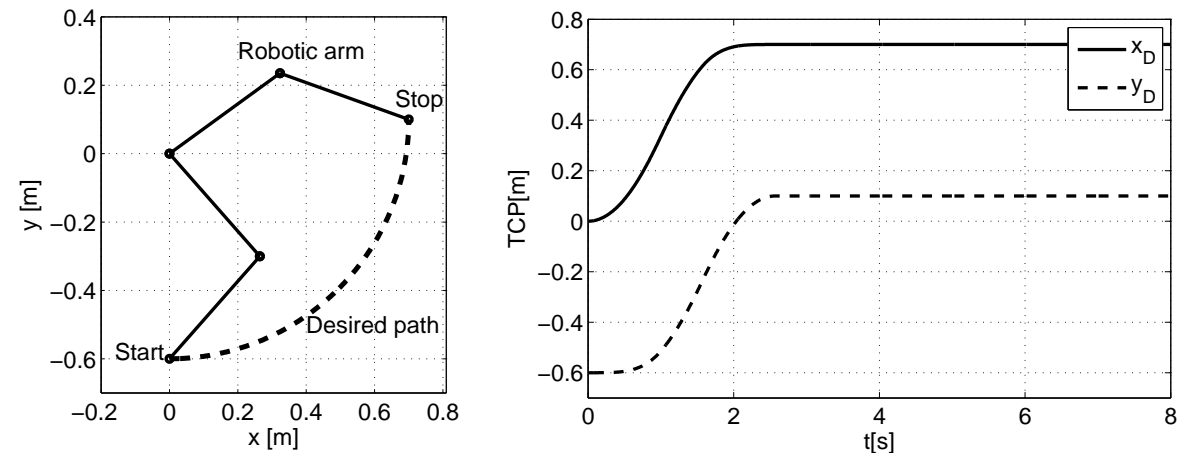

Figure 3: Desired path (left) and corresponding TCP trajectories (right)

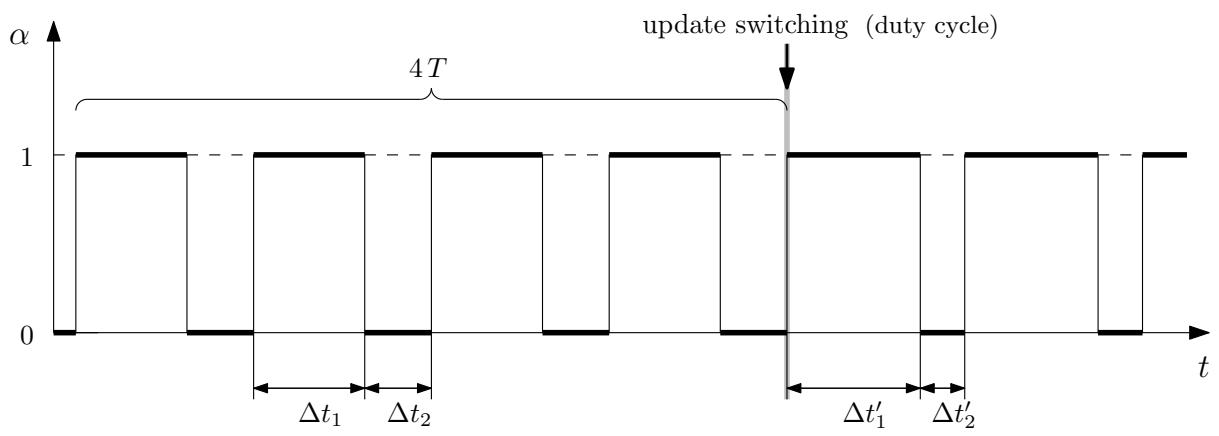

Figure 4: Switching function for periodic servo-constraint realization

where now $\widehat{\gamma}_{i} \equiv \gamma_{i}, i=1,2$, and the periodic function $\alpha(t)=\alpha(t+T)$ controls the switching between these servo-constraints. In the current example, this is realized such that each period include 20 integration time steps $(h=0.01 \mathrm{~s})$, and the duty cycle of the switching function $\alpha(t)$ is updated after every 4th period as it is illustrated in Fig. 4. Note, that the update frequency is a free control parameter which needs to be tuned by simulation or can be selected empirically. The switching between the control objectives represented by each reduced set of servo constraints is however algorithmically defined by equation (14). For the two-link manipulator example, this 
equation gives the duty cycle parameters, $\Delta t_{1}$ and $\Delta t_{2}=T-\Delta t_{1}$, as follows

$$
\Delta t_{i}=\frac{z_{i} T}{z_{1}+z_{2}}, i=1,2 \quad \text { with } \quad z_{1}=\frac{b_{21}^{2}+b_{22}^{2}}{\left|b_{21} \bar{m}_{11}+b_{2} 2 \bar{m}_{21}\right|} \quad \text { and } \quad z_{2}=\frac{b_{21}^{2}+b_{22}^{2}}{\left|b_{21} \bar{m}_{12}+b_{22} \bar{m}_{22}\right|}
$$

where $z_{i}=\left(\mathbf{e}_{i}^{\top} \mathcal{M} \mathbf{e}_{i}\right)^{-1 / 2}$, and unit vectors $\mathbf{e}_{1}=[1,0]^{\top}$ and $\mathbf{e}_{2}=[0,1]^{\top}$ represent the different servo-constraint directions.

When there is no actuator saturation, the required control input can be determined based on equations (7) and (16) by disregarding the unnecessary geometric constraints. During saturation, equations (9) has to be considered with the terms defined in equations (15) and (16), and using the periodic servo-constraints given by equations (17) and (18). Simulation results obtained with this proposed control scheme are compared to those of the classical computed torque control in Fig. 5. It can be seen that both methods have simultaneous, but different magnitude, peaks in the constraint violations. The periodic controller seems to outperform the other in the second half of the simulation, and the system recovers from the saturation slightly faster when periodic servo-constraints are applied (see $\tau_{1}$ in Fig. 5). When the norm of the servo-constraint violation is considered, the better performance of the periodic controller is obvious. This is shown in Fig. 6 where the results obtained with two other methods available in the literature are also presented. The method proposed in [10] is similar to ours in the sense that when saturation happens a reduced set of servo-constraint is constructed, which is then used until the system recovers from saturation. The main difference between this and our approach is the periodic variation of different reduced sets of servo-constraint as opposed to using only one. Also, in the present paper we propose an algorithmic approach to select to which extent the different sets of servo-constraints, representing different control objectives, are considered in the periodic control signal. In Fig. 6 it is shown that the two methods perform similarly in the beginning and at the end of the simulated motion, but the use of periodic servo-constraints could considerably reduce the trajectory tracking error in the middle. This is the part of the trajectory which changes the most (see Fig. 3). We also note that the results 
are directly comparable as [10] does not require any additional control parameters to be defined.

As a second method to compare with, let us consider the anti-windup control scheme presented in [8].

Among the many general purpose anti-windup schemes, this one is specifically developed for

Euler-Lagrange systems. It is well suited for robotic mechanical systems, which makes the comparison clearer. For the implementation of the method, in the present example, three additional

control parameters had to be chosen. By keeping the notations of the referenced paper, these anti-windup design parameters are $K_{g}=0.99, K_{q}=10$ and $K_{0}=1$. Parameter $K_{g}$ is selected to the same value used in [8] in a similar example, while the PD type control gains $K_{q}$ and $K_{0}$ were tuned empirically to achieve a nearly optimal performance. This method and the use of periodic servo-constraints results in similar results almost in the entire course of the motion, but the periodic controller shows a much better convergence to the desired zero steady state error.

When the tracking error of the four different methods are compared, Fig. 6 suggest that the best results can be achieved with the method of periodic servo-constraints. This is confirmed by the RMS (root-mean-square) values presented in Tab. 1. It is clearly shown that the methods in [10] and [8] has a very similar performance, while the method of periodic-servo constraints gives both the lowest peak - and average tracking errors. With respect to the use of the original servo-constraints, and the other two methods, the average tracking performance is improved by $19 \%$ and $21 \%$, respectively.

\section{CONCLUSIONS}

There are three main contributions in this work. The first is the systematic introduction of periodic servo-constraints for the control of temporarily underactuated, saturated robotic systems. The proposed method successfully combine the stabilizing effect of periodic controllers with the need of satisfying, and prioritizing between, different control objectives.

The second contribution is the introduction of a new, manipulability type performance measure for the algorithmic selection of a suitable periodic control signal. The proposed method is generally 

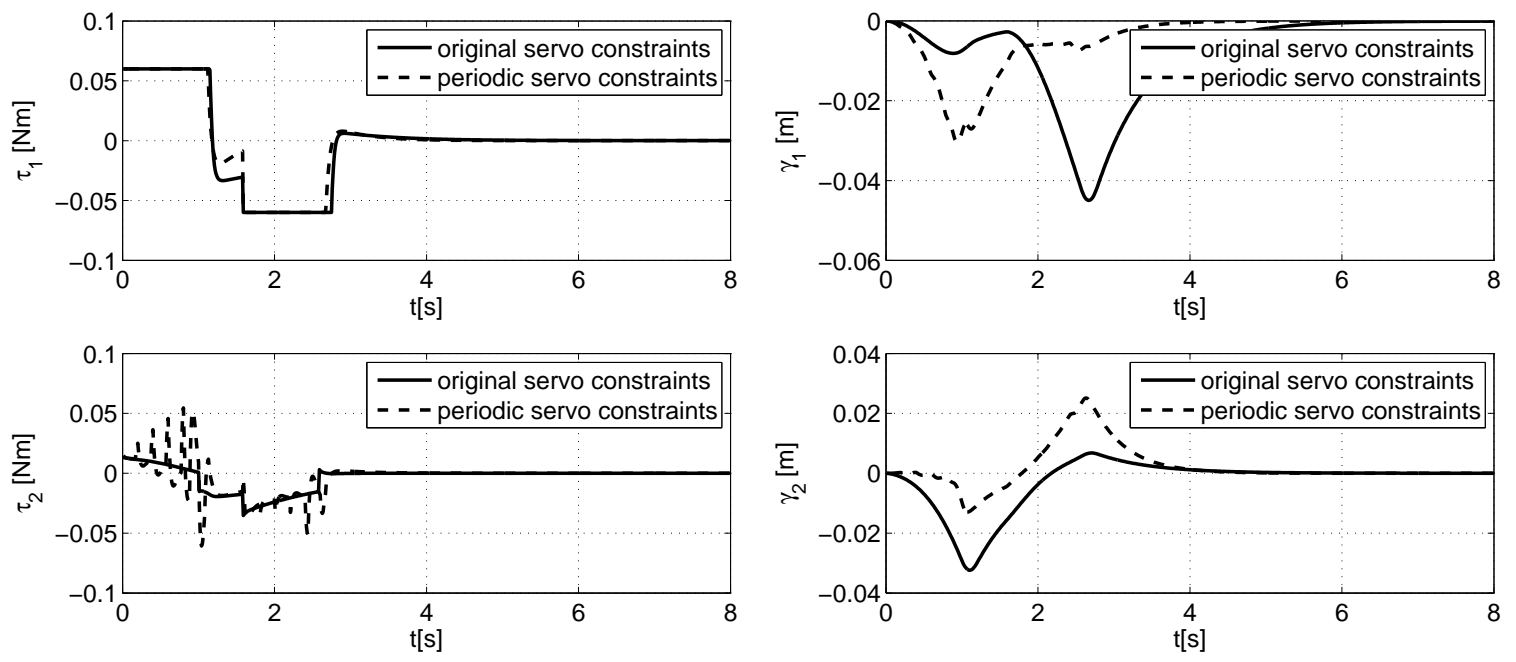

Figure 5: Actuator efforts (left) and servo-constraint violations

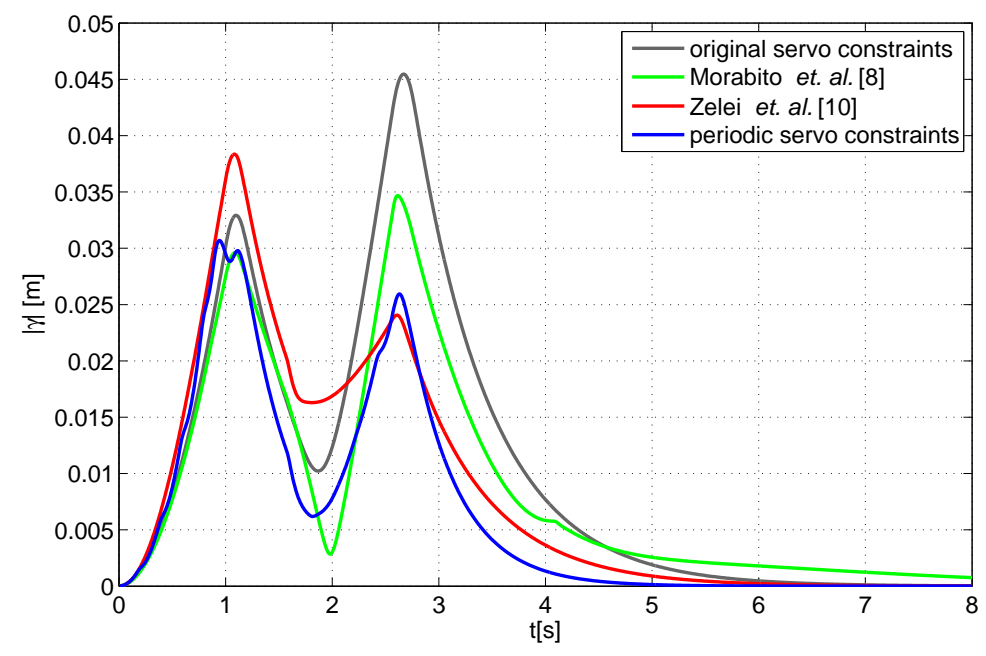

Figure 6: Comparison of different methods using the norm of servo-constraint violations 
Table 1: Peak and RMS trajectory tracking errors

\begin{tabular}{lcc} 
Control method & $\max \gamma[m]$ & $\sqrt{\frac{1}{n} \sum_{i=1}^{n} \gamma\left(t_{i}\right)^{2}}[m]$ \\
\hline Original servo-constraints & 0.046 & 0.0164 \\
Morabito et. al. $[8]$ & 0.034 & 0.0130 \\
Zelei et. al. $[10]$ & 0.038 & 0.0134 \\
Periodic servo-constraints & 0.031 & 0.0119 \\
\hline
\end{tabular}

applicable to wide class of robotic systems, but it is still easy to implement, and it was proved to be effective in achieving better tracking performance and faster convergence to the steady state error than other methods taken from the literature.

The detailed comparison with the other methods can be considered as the third contribution. The peak - and average tracking errors were contrasted in a benchmark example. The results are promising, as even a relatively simple periodic switching strategy could considerably decrease the negative effects of actuator saturation. Future work include the investigation of the effect of using different dynamic performance measures, e.g., effective inertia, and optimizing the currently free control parameters such as the time and update periods of the periodic servo-constraints.

\section{Acknowledgement}

This research has been supported by the MTA-BME Research Group on Dynamics of Machines and Vehicles. This support is gratefully acknowledged.

\section{Conflicts of Interest Statement}

The authors have NO affiliations with or involvement in any organization or entity with any financial interest (such as honoraria; educational grants; participation in speakers' bureaus; membership, employment, consultancies, stock ownership, or other equity interest; and expert testimony or 
patent-licensing arrangements), or non-financial interest (such as personal or professional relationships, affiliations, knowledge or beliefs) in the subject matter or materials discussed in this manuscript. 


\section{References}

[1] Zelinsky A., Field and Service Robotics, Springer-Verlag, London, 1998.

[2] Galeani S., Tarbouriech S., Turner M. et al., A tutorial on modern anti-windup design, in Proceedings of the European Control Conference, 306-323, Budapest, Hungary, August 23-26, 2009.

[3] Doyle J., Smith R. and Enns D., Control of plants with input saturation nonlinearities, in Proceedings of Americal Control Conference, 1034-1039, 1987.

[4] B. Bequette W., Process Control: Modeling, Design, and Simulation, Prentice Hall Professional, New Jersey, 2003.

[5] Sung-Uk L. and Hun C.P., The Development of Anti-Windup Scheme for Time Delay Control With Switching Action Using Integral Sliding Surface, Journal of Dynamic Systems, Measurement, and Control, 125(4):630-638, 2004.

[6] Kapoor A., Teel A.R. and Daoutidis P., An Anti-Windup Design for Linear Systems with Input Saturation, Automatica, 34(5):559-574, 1998.

[7] A. Kendi T. and Doyle F.J., An anti-windup scheme for multivariable nonlinear systems, Journal of Process Control, 7(5):329-343, 1997.

[8] Morabito F., Teel A.R. and Zaccarian L., Nonlinear Antiwindup Applied to Euler-Lagrange Systems, IEEE Transactions on Robotics and Automation, 20(3):526-537, 2004.

[9] Naghshineh M. and M. K., Actuator Saturation Avoidance in Overactuated Systems, in Proceedings of 2004 IEEE/RSJ International Conference on Intelligent Robots and Systems, 3613-3618, Sendai, Japan, September 28 - October 2, 2004. 
[10] Zelei A. and Stépán G., Computed torque control of a constrained manipulator considering the actuator saturation, in Proceedings of 11th Conference on Dynamical Systems - Theory and Applications, 184-194, Lodz, Poland, December 5-8, 2011.

[11] Blajer W., Seifried R. and Kołodziejczyk K., Diversity of Servo-Constraint Problems for Underactuated Mechanical Systems: A Case Study Illustration, Solid State Phenomen, 198:473-482, 2013.

[12] Seifried R., Dynamics of underactuated multibody systems: Modeling, Control and Optimal Design, Solid Mechanics and Its Applications, 205, 2014.

[13] Kovács L.L. and Bencsik L., Stability case study of the ACROBOTER underactuated service robot, Theoretical and Applied Mechanics Letters, 2(4), 2012.

[14] Bencsik L., Kovács L.L. and Zelei A., Periodic Servo-Constraints for Stabilizing Underactuated Multibody Systems, in Book of Absrtacts of The 2nd Joint International Conference on Multibody System Dynamics-IMSD2014, Busan, Korea, June 30-July 3, 2014.

[15] de Jalón J. and Bayo E., Kinematic and dynamic simulation of multibody systems: the real-time challenge, Springer-Verlag, New York, 1994.

[16] Kirgetov V.I., The Motion of Controlled Mechanical Systems with Prescribed Constraints (Servo Constraints), Prikl. Mat. Mekh., 31(3):433-447, 1967.

[17] Baumgarte J., Stabilization of constraints and integrals of motion in dyanamical systems, Computer methods in applied mechanics and engineering, 1(1):1-16, 1972.

[18] Isidori A., Nonlinear Control Systems, 3rd edition, Springer-Verlag, London, 1995.

[19] Insperger T. and Stépán G., Act-and-wait control concept for discrete-time systems with feedback delay, IET Control Theory \& Applications, 1(3):553-557, 2007. 
[20] Khatib O., Inertial Properties in Robotic Manipulation, The International Journal of Robotics Research, 13(1):19-36, 1995. 